\title{
ANSIEDAD Y MIEDO ANTE EL TRATAMIENTO ODONTOLÓGICO EN NIÑOS
}

\section{ANXIETY AND FEAR ABOUT DENTAL TREATMENT IN CHILDREN}

\author{
Ledesma-Herrera Kimberlyn ${ }^{1}{ }^{*}$ Villavicencio-Caparó Ebingen ${ }^{2}$ \\ ${ }^{1}$ Odontóloga Universidad Católica de Cuenca. Ecuador. \\ ${ }^{2}$ Catedrático de la Carrera de Odontología de la Universidad Católica de Cuenca. Ecuador. \\ *kimylh.95@gmail.com
}

\begin{abstract}
Resumen
Se realizó una revisión bibliográfica cuyo objetivo era recolectar información científica de distintos autores en todos los ámbitos sobre ansiedad y miedo ante el tratamiento odontológico con el propósito de obtener datos estadísticos respecto a ansiedad y miedo dental como una respuesta habitual hacia el tratamiento dental que va avanzando progresivamente y que puede interferir en la relación odontólogo-paciente y en la calidad de vida del niño; se analizó especificamente información que estudiaba este fenómeno en población infantil y se pudo obtener datos importantes sobre los factores de riesgo entre ellos vibración o perforación de las fresas; se proporciona información sobre las escalas de valoración más eficaces y utilizadas por los especialistas, además de dar a conocer los métodos de manejo al paciente pediátrico y tratamiento del paciente ansioso y temeroso, siendo el más destacado la musicoterapia. Conclusión: El miedo y la ansiedad son dos fenómenos que van de la mano y que han ido progresando con el tiempo; ya que los profesionales no le han dado la importancia necesaria para tratar este problema, esta reacción ha ido empeorando causando una serie de problemas futuros en los pacientes pediátricos.
\end{abstract}

Palabras clave: Ansiedad al tratamiento odontológico, miedo al tratamiento odontológico, Odontología Pediátrica.

\begin{abstract}
A bibliographic review was conducted to collect scientific information from different authors in all areas on anxiety and fear of dental treatment in order to obtain statistical data regarding anxiety and dental fear as a habitual response to dental treatment that is progressing Progressively and that can interfere in the relation dentist-patient and in the quality of life of the child; The information that studied the phenomenon was analyzed specifically in the child population and it was possible to obtain important data on the risk factors among them vibration or drilling of the strawberries; The information about the most effective and used scales of evaluation by specialists, as well as the methods of management of the pediatric patient and the treatment of the anxious and fearful patient, being the most outstanding music. Conclusion: Fear and anxiety are phenomena that go hand in hand and have been progressing over time; Since professionals have not given it enough importance to address this problem, this reaction has worsened causing a number of future problems in pediatric patients.
\end{abstract}

Key words: Dental anxiety, Dental fears, Pediatrics dentistry.

\section{INTRODUCCIÓN}

La ansiedad y el miedo son dos problemas comunes que los odontólogos pediátricos se encuentran a diario, puede afectar en el acceso y la demanda de servicios de salud dental, también en el manejo del paciente e intervenir en la efectividad del tratamiento, dando lugar a distintas consecuencias, esta puede persistir en la edad adulta formando una barrera entre el profesional y el paciente. De ahí radica la importancia en que los niños clínicamente ansiosos se identifiquen lo antes posible y se les proporcione intervenciones apropiadas. $^{1,2}$

La ansiedad se entiende como una respuesta a circunstancias en las cuales el origen de la amenaza para la persona no está bien comprendida o reconocida, es la anticipación de un peligro indefinible; se refiere a un estado emocional que tiene su origen en el interior de la persona relacionándolo con fantasías y expectativas no existentes; es difícil aislar totalmente el temor de la ansiedad, ya que este nunca actúa sin un cierto grado de ansiedad porque "es temor a lo desconocido. ${ }^{3,4}$ 
La visita al dentista causara un cierto grado de ansiedad, en casi todos los niños, y ellos expresan su conducta de acuerdo con su edad y su nivel de discernimiento o madurez emocional, especialmente en preescolar, hasta la pubertad, se convierte en una percepción más profunda por parte del paciente que interfiere con el tratamiento habitual y requiere una atención especial. ${ }^{4,5}$

El miedo es la respuesta hacia una situación donde un individuo se siente amenazado, es un estado emocional del sujeto que se caracteriza por un profundo sentimiento, generalmente incómodo y desagradable, provocado por la percepción de un peligro, existente o aparente, presente o futuro, ${ }^{6,7}$ es una alteración que expresa temor y que tiende a producir actuaciones de huida o escape e invade y afecta al paciente tanto en su entorno social como a nivel familiar, laboral y/o en el estudio. , $^{7}$

Es de gran relevancia saber identificar los niveles de ansiedad y miedo de los pacientes en el área odontopediátrica, así como su comportamiento durante los distintos procedimientos dentales, ya que este fenómeno tiene la capacidad de formar una barrera para que el profesional no realice su trabajo con éxito. ${ }^{9}$ Cuando el profesional de salud intenta manejar las reacciones de los pacientes causados por la ansiedad y resulta complicado manejar estos comportamientos, se convierte en fuentes que producen estrés para el odontólogo y para los pacientes. ${ }^{10}$

El miedo y la ansiedad se pueden diferenciar porque el miedo actúa como un sentimiento que reacciona ante peligros que están pasando ese momento y son fáciles de identificar mientras que la ansiedad se describe como un estado emocional que reacciona a una amenaza irreconocible, es decir no está bien definida o esperada. ${ }^{11}$

\section{FACTORES DE RIESGO DE ANSIEDAD Y MIEDO ANTE EL TRATAMIENTO ODONTOLÓGICO}

Existen diferentes factores de riesgo que podrían desencadenar niveles de miedo y ansiedad; pudiendo ser estos los más relevantes.

Albuquerque en el 2007 en su artículo expresa que hay tres factores desencadenantes de ansiedad y miedo: novedad, inseguridad y expectativa; es decir los pacientes que acuden a la consulta por primera vez desconocen del procedimiento que se les va a realizar y se dejan llevar por comentarios de su entorno familiar y social con malas experiencias previas generando inseguridad en el paciente. ${ }^{3}$

Hmud R en el año 2009 concluyó que hay varios factores que intervienen y modifican la ansiedad y el miedo en la consulta odontológica clasificándolos de la siguiente manera (Tabla 1)

En los análisis verificados de la población infantil, nos pudieron revelar que los niños tienen más miedo o ansiedad ante tratamientos dentales que sean más invasivos y dolorosos: $:^{10,12}$ (Ver la tabla 2)

En la Tabla 3 podemos observar los factores de riesgo que causan ansiedad y miedo en la consulta odontológica, los que predominan según el estudio de Moore en el año 1993 es la sensación de vibración con las fresas con un $\mathrm{OR}=38.7$ siendo el principal factor de riesgo, seguido de la actitud o comportamiento del dentista o el personal y el procedimiento de profilaxis dental, esto generalmente en niños según Hmud; Moore en el 2001 complementa su información mencionando que los problemas psicológicos son un factor de riesgo significativo que incrementa la ansiedad y el miedo al odontólogo.

Al hacer una recopilación de la información Caycedo, Hmud y Rivera mencionan que los procedimientos dentales que inducen náuseas, sensación de ahogo o sofoco generan más ansiedad; al mismo tiempo el trato con extraños, es decir, la separación de los padres durante el tratamiento de sus niños de corta edad hace que tengan que pasar por experiencias traumáticas que podrían afectar en el futuro . ${ }^{10,12,21}$

Rivera en el 2005 asegura en su estudio que pasar por una mala experiencia por primera vez en la consulta odontológica sería un factor de riesgo significativo que corrobora a que el niño tenga ansiedad en el futuro, ya que su investigación aseguro que los niños que habían tenido experiencias traumáticas pasadas o experiencias en las cuales experimentaban dolor eran más ansiosos que los niños que no las habían pasado. ${ }^{12}$

Diferentes investigaciones han demostrado que el miedo y la ansiedad tienen un origen multifactorial donde el dolor, experiencias negativas, la madurez, timidez, educación del niño, y la falta de autoridad de los padres serían las principales causas que producen estos comportamientos ${ }^{9}$ hace que afecten en forma negativa la salud bucodental gracias a la falta de atención profesional por miedo del niño a ser tratado.

\section{FRECUENCIA DE ANSIEDAD Y MIEDO EN EL TRATAMIENTO ODONTOLÓGICO EN NIÑOS}

Se considera que el $10 \%$ y el $15 \%$ de la población mundial tienen algún grado de ansiedad y miedo al dentista, esto provoca la cancelación y postergación de citas al odontólogo interfiriendo con la salud bucal del paciente; se estima que tiene una prevalencia $4 \%$ al $23 \%$ por eso hay que tener en cuenta que la ansiedad es un fenómeno que debemos tratar a tiempo si es que queremos mejorar la calidad de vida del paciente. ${ }^{22}$

Estudios epidemiológicos sugieren que entre el 3\% y el $20 \%$ de la población tiene niveles de miedo y Ansiedad 
Tabla 1. Factores de Riesgo de ansiedad y miedo en la consulta odontológica Hmud R, (2009)

\begin{tabular}{llll}
\hline PACIENTE & DENTISTA & PROCEDIMIENTO & \multicolumn{1}{c}{ LUGAR } \\
\hline $\begin{array}{l}\text { Características de } \\
\text { personalidad }\end{array}$ & $\begin{array}{l}\text { Técnicas/aptitudes de } \\
\text { comunicación }\end{array}$ & $\begin{array}{l}\text { Anestesia (miedo a las } \\
\text { agujas) }\end{array}$ & Sonidos de fresas \\
$\begin{array}{l}\text { Experiencias dentales } \\
\text { traumáticas }\end{array}$ & $\begin{array}{l}\text { Profesional o personal } \\
\text { mal humorados }\end{array}$ & $\begin{array}{l}\text { Sensación de vibración } \\
\text { y rotación de fresas }\end{array}$ & Olores \\
$\begin{array}{l}\text { Aprendizaje vicario } \\
\text { (relatos de terceros) }\end{array}$ & $\begin{array}{l}\text { Personal poco } \\
\text { comprensivo }\end{array}$ & Extracción & $\begin{array}{l}\text { Sala de } \\
\text { espera(distribución o } \\
\text { diseño) }\end{array}$ \\
$\begin{array}{l}\text { Temor al dolor } \\
\text { Temor a la sangre y } \\
\text { heridas }\end{array}$ & $\begin{array}{l}\text { negativo del equipo } \\
\text { dental. }\end{array}$ & Profilaxis & \\
$\begin{array}{l}\text { Desordenes } \\
\text { psicológicos previos }\end{array}$ & & $\begin{array}{l}\text { Tratamiento de } \\
\text { conducto }\end{array}$ & $\begin{array}{l}\text { Ruidos o quejas } \\
\text { provenientes de } \\
\text { pacientes }\end{array}$ \\
\hline
\end{tabular}

Tabla 2. Factores de Riesgo de ansiedad y miedo ante el tratamiento odontológico

\begin{tabular}{|c|c|c|c|}
\hline \multicolumn{4}{|c|}{ Factores de Riesgo y causas más frecuentes de Ansiedad y Miedo al Odontólogo } \\
\hline Referencia & Características & $\mathbf{P}$ & Factor de Riesgo \\
\hline Doeer P. $1998^{(13)}$ & Sexo & $<0.00$ & (FR) significativo \\
\hline Doeer P. 1998 & Raza & 0.43 & (FR) No significativo \\
\hline Doeer P. 1998 & Consultas frecuentes & $<0.10$ & (FR) No significativo \\
\hline Doeer P. 1998 & Mismo Lugar para atenderse & $<0.00$ & (FR) significativo \\
\hline Doeer P. 1998 & Actitud del dentista o personal & $<0.00$ & (FR) significativo \\
\hline Moore R. $2001^{(14)}$ & Miedo al dolor & $<0.05$ & (FR) significativo \\
\hline Moore R. 1993 (15) & Miedo a las agujas & 0.99 & (FR) No significativo \\
\hline Moore R.1993 & Profilaxis & 0.01 & (FR) significativo \\
\hline Moore R.1993 & Actitud del dentista o personal & 0.02 & (FR) significativo \\
\hline Moore R.1993 & Sexo femenino & 0.11 & (FR) No significativo \\
\hline Moore R. 1993 & Altos Ingresos & 0.48 & (FR) No significativo \\
\hline Moore R.1993 & $\begin{array}{l}\text { Sensación de vibración o } \\
\text { perforación }\end{array}$ & 0.00 & (FR) significativo \\
\hline Mc Neil.2011 (16) & $\begin{array}{l}\text { Experiencia de extracción } \\
\text { traumática pasada }\end{array}$ & $<0.05$ & (FR) significativo \\
\hline Crofts. N $2010^{(17)}$ & Calidad de vida como dormir & 0.01 & (FR) significativo \\
\hline Crofts.N 2010 & Factores psicológicos & 0.05 & $(F R)$ significativo \\
\hline
\end{tabular}

acerca del tratamiento dental que puede considerarse problemática. ${ }^{23}$

El porcentaje de niños con altos grados de ansiedad puede variar, todo depende de las características de la población estudiada, tomando en cuenta la edad, población en general o clínica, desordenes psicológicos y primeras visitas al odontólogo o no, etc. ${ }^{12}$ (Ver la Tabla 5-6.)

La edad es un factor el cual se encuentra relacionado con los niveles de ansiedad y miedo en los niños; estudios han demostrado y comprobado que los niños más pequeños manifiestan niveles de ansiedad más altos que los niños de mayores esto confirma el estudio de Ramos, Tazaico y Rivera en 2013; 2010 y 2005 respectivamente en sus investigaciones comprobaron que los niños de 5 a 7 años tuvieron más prevalencia de ansiedad que los mayores de 8 años. ${ }^{6,12,28}$ 
Tabla 3. Factores de Riesgo de ansiedad y miedo ante el tratamiento odontológico. valores OR

\begin{tabular}{|c|c|c|c|c|}
\hline Referencia & Características & OR & IC al $95 \%$ & FACTOR DE RIESGO \\
\hline Arrieta. 2013 & $\begin{array}{l}\text { Experiencia } \\
\text { traumática previa }\end{array}$ & 3.2 & $1,7-6,3$ & Representa (FR) \\
\hline Moore R. $2001^{(14)}$ & $\begin{array}{l}\text { Problemas } \\
\text { Psicológicos }\end{array}$ & 2.4 & $0,8-7,1$ & Representa (FR) \\
\hline Moore R. 2001 & Estrés del dentista & 2.2 & $0,8-6,3$ & Representa (FR) \\
\hline Moore R. 2001 & Miedo al dolor & 1.9 & $0,7-5,5$ & Representa (FR) \\
\hline Elter J. $1997^{(18)}$ & $\begin{array}{l}\text { Experiencias } \\
\text { traumática previa }\end{array}$ & 2.1 & $1,2-3,6$ & Representa (FR) \\
\hline Moore R. 1993 (15) & Miedo a las agujas & 1.0 & $0,2-4,8$ & No representa (FR) \\
\hline Moore R.1993 & Profilaxis & 7.3 & $1,5-36,8$ & Representa (FR) \\
\hline Moore R.1993 & $\begin{array}{l}\text { Actitud del dentista } \\
\text { o personal }\end{array}$ & 9.3 & $1,4-60,7$ & Representa (FR) \\
\hline Moore R.1993 & Sexo femenino & 4.2 & $0,7-24,7$ & Representa (FR) \\
\hline Moore R. 1993 & $\begin{array}{l}\text { Altos Ingresos } \\
\text { Sensación de }\end{array}$ & 1.8 & $0,4-9,4$ & No representa (FR) \\
\hline Moore R.1993 & $\begin{array}{l}\text { vibración o } \\
\text { perforación }\end{array}$ & 38.7 & $0,1-2,2$ & Representa (FR) \\
\hline
\end{tabular}

Tabla 4. factores de riesgo y causas mas frecuentes de Ansiedad y Miedo al odontólogo, porcentajes.

\begin{tabular}{lcc}
\hline \multicolumn{1}{c}{ Referencia } & Características & $\%$ \\
\hline Moore R. 2001 (14) & Miedo al dolor & $44.4 \%$ \\
Moore R. 2001 & Experiencias traumáticas pasadas & $50.9 \%$ \\
Moore R. 2001 & Problemas Psicológicos & $38.5 \%$ \\
Moore R. 2001 & Vergüenza del estado de salud Oral & $32.3 \%$ \\
Moore R. 2001 & Aprendizaje vicario & $14.9 \%$ \\
Moore R. 2001 & Problemas Económicos & $1.9 \%$ \\
Lima M.2008 ${ }^{(19)}$ & Impresión producida por el instrumental & $74.19 \%$ \\
Lima M.2008 & Ruido de equipos & $54.84 \%$ \\
Lima M.2008 & Experiencia traumáticas pasadas & $48.39 \%$ \\
Rav, J. $2010^{(20)}$ & Factores genéticos & $8.5 \%$ \\
\hline
\end{tabular}

4 MÉTODOS DE DIAGNÓSTICO DE ANSIEDAD Y MIEDO ANTE EL TRATAMIENTO ODONTOLÓGICO

Para medir la ansiedad y el miedo existen distintos instrumentos llamados escalas específicamente diseñadas para medir el nivel de miedo y ansiedad que tiene el paciente; estos se encuentran ampliamente citados en la literatura, son válidos y confiables y actúan como un método auxiliar para que el profesional prevenga el posible comportamiento en la consulta odontológica. ${ }^{3}$

La escala Dental Fear Survey (DFS), dental Cope
Questionnaire (DCQ) y Escala de evaluación del miedo dental en niños (CFSS-DS) son los principales instrumentos utilizados para evaluar el nivel de miedo al llegar a la consulta odontológica, siendo este último el más usado en niños. La ansiedad se la puede evaluar de distintas formas, pero la forma más útil es aplicando los siguientes test que nos dan una valoración exacta del nivel exacto que podría tener el paciente, la escala de Corah (CDAS) es considerada una escala de oro para valorar la ansiedad al odontólogo junto con la (MDAS) que es una modificación de la escala de corah; la escala de Venham (VPT) y Facial Imagen Scale (FIS) son las escalas más consideradas para valorar la 
Tabla 5. Frecuencia de ansiedad en niños medida con distintos instrumentos.

\begin{tabular}{|c|c|c|c|c|}
\hline Referencia & País & Instrumento & Edad & $\begin{array}{l}\% \text { De niños con } \\
\text { ansiedad }\end{array}$ \\
\hline \multirow[t]{3}{*}{ Lazo A.2010 (5) } & Perú & FIS (Facial Imaqe scale) & 4 a 8 años & $38 \% \operatorname{con}(\mathrm{AM})$ \\
\hline & & & & $56 \%$ con $(A L) ; 26 \%$ \\
\hline & & & & con (AM); $11 \%$ con \\
\hline \multirow[t]{2}{*}{ Coric, A.2014 (24) } & EEUU & C'DAS (Corah modificada) & 7-15 años & (AS) \\
\hline & & MFAS (Modified Facial Affective & & $44 \%$ con (AM); $11 \%$ \\
\hline Tiwari, N.2015(25) & India & Scale) & 6-8 años & $\operatorname{con}(A S)$ \\
\hline \multirow[t]{3}{*}{ Ramos, K. $2013^{(6)}$} & Colombia & FIS -C'DAS & 3-8 años & $13,44 \%$ con (AS) \\
\hline & & & & $31,03 \%$ con $(A L)$ \\
\hline & & & & $13.79 \%(\mathrm{AM})$ \\
\hline \multirow[t]{2}{*}{ Ciraiz E.2015 (26) } & Guatemala & VPT (Venham) & 5-9 años & $1.72 \%(A S)$ \\
\hline & & & & $65 \% \operatorname{con}(A L) ; 24 \%-$ \\
\hline Luna, G.2015(27) & Ecuador & C'DAS (Corah modificada) & 4-14 años & $(\mathrm{AM})$ \\
\hline
\end{tabular}

AM: Ansiedad Moderada; AL: Ansiedad Leve; AS: Ansiedad Severa

Tabla 6. Frecuencia de miedo en niños medida con distintos instrumentos

\begin{tabular}{|c|c|c|c|c|}
\hline Referencia & País & Instrumento & Edad & $\begin{array}{l}\text { Frecuencia de niños con } \\
\text { Miedo }\end{array}$ \\
\hline & & CFSS (Children Fear & $6-11$ & $84,1 \%$ con $\mathrm{ML}$ y $\mathrm{MM} 27 \%$ \\
\hline \multirow[t]{2}{*}{ Rivera. $2005^{(12)}$} & Honduras & Survey Scale) & años & con MS \\
\hline & & CFSS (Children Fear & $7-15$ & \\
\hline \multirow[t]{2}{*}{ Coric. $20144^{(24)}$} & EEUU & Survey Scale) & años & $14 \%$ con $\mathrm{MM} ; 11 \%$ con $\mathrm{MS}$ \\
\hline & & CDFD(Children Dental & $6-8$ & \\
\hline \multirow[t]{2}{*}{ Tiwari. $2015^{(25)}$} & India & Picture Test) & años & $44.4 \%$ \\
\hline & & CFSS (Children Fear & 4-14 & $34 \%$ con $\mathrm{MM} ; 42.5 \%$ con \\
\hline \multirow[t]{2}{*}{ Sunil. $2013^{(29)}$} & India & Survey Scale) & años & MS \\
\hline & & CFSS (Children Fear & $3-8$ & \\
\hline Ramos $.2013^{(6)}$ & Colombia & Survey Scale) & años & $18,12 \% \mathrm{MS}$ \\
\hline
\end{tabular}

ML:Miedo Leve; MM: Miedo Moderado; MS: Miedo Severo

ansiedad en niños pequeños. ${ }^{9,30}$

Haciendo una comparación en la literatura, se puede decir que las escalas más utilizadas, válidas y confiables para la valoración de la ansiedad, las escalas FIS, VPT, C DAS (con sus limitaciones); para la valoración del miedo dental, debido a su éxito y buenos resultados, la CFSS-DS. ${ }^{9}$ ( Ver Tabla 7.)

4.1 Tratamiento y manejo del paciente con miedo y ansiedad al Odontólogo.

Para solucionar este fenómeno de la ansiedad y miedo ante el tratamiento odontológico, diferentes autores han descrito en este último tiempo estrategias básicas que se han implementado para poder disminuir estos síntomas durante la consulta dental y mejorar la relación odontólogo-paciente. ${ }^{22}$

Hay varios tratamientos que se pueden seguir, pero todo depende del nivel de ansiedad que adquirió el niño en su tiempo pasado, generalmente es importante evaluar la conducta a seguir de cada paciente; ya que si no se trata este fenómeno a tiempo, el niño corre el riesgo de tener problemas de salud generales graves, interferir su calidad de vida, además de afectar de gran forma a la salud oral por la inasistencia a sus citas, así como a la eficacia del tratamiento odontológico recibido; es un factor importante 
Tabla 7. Instrumentos más utilizados para medir ansiedad y miedo al odontólogo.

\begin{tabular}{|c|c|c|c|c|c|}
\hline Referencia & Escala & validez & Confiabilidad & $\begin{array}{l}\text { Gold } \\
\text { estándar }\end{array}$ & $\begin{array}{l}\text { Cronbach alpha } \\
\text { valores }\end{array}$ \\
\hline Singh $(2010)^{(30)}$ & CFSS-DS & & 0,92 & & 0,92 \\
\hline Howard (2007) $)^{(31)}$ & MCDAS & 0,82 & 0,8 & CFSS-DS & 0,82 \\
\hline Buchanan.H(2002) ${ }^{(32)}$ & FIS & 0,7 & & VPT & \\
\hline Bahammam (2014) ${ }^{(33)}$ & MCDAS & 0,9 & & VAS & 0,9 \\
\hline Lalić M,(2015) ${ }^{(34)}$ & CFSS-DS & 0,88 & & CDFQ & 0,88 \\
\hline Tunc. EP (2005) $)^{(35)}$ & MDAS & $\begin{array}{l}0,80 \\
\text { sensibilidad }\end{array}$ & $\begin{array}{l}0,74 \\
\text { especificidad }\end{array}$ & DFS & \\
\hline Maistorovic.M (2003) ${ }^{(36)}$ & CDAS & & & & 0,78 \\
\hline Maistorovic.M (2003) & CFSS-DS & & & & 0,83 \\
\hline Maistorovic.M (2003) & S-DAI & 0,66 & 0,66 & CDAS & 0,86 \\
\hline \multicolumn{6}{|l|}{ Lin. C. $(2017)^{(37)}$} \\
\hline & STAI & & 0.89 & VAS & 0,89 \\
\hline Lin. C (2017) & MDAS & & 0.88 & CDAS & 0.89 \\
\hline Lin.C (2017) & SDAI & & 0.88 & DFS & 0.88 \\
\hline
\end{tabular}

a considerar si se quiere mejorar la calidad de vida oral del paciente. $^{12,22,38}$

Caycedo en el 2008, manifiesta que para disminuir la ansiedad y miedo de los pacientes se puede recurrir a tratamientos de tipo farmacológico como la sedación intravenosa, oral, psicoterapia. Otros autores manifiestan la hipnosis como buen resultado pero se requiere de citas previas para su preparación. ${ }^{10,22}$

Hmud en el 2004, manifiesta que maneras de prevenir la ansiedad podrían ser: dar suficiente tiempo para la cita dental, minimizar los factores provocadores, introducir métodos de relajamiento como la musicaterapia, usar técnicas de distracción y remitir al paciente a especialistas que manejen el ámbito psicológico. ${ }^{21}$

Bare y Dundes en el 2004, indicaron que los medios de distracción son eficaces para el manejo de la ansiedad y miedo al odontólogo, como utilizar música de fondo en el consultorio en un $89 \%$ los ayudaban a relajarse; mientras que un $75 \%$ consideró que la presencia de revistas y libros reducía la ansiedad. ${ }^{39}$ (Ver Tabla.8)

Lima en el 2008 aplicó técnicas de relajación en sus pacientes para demostrar el control de la ansiedad y pudo comprobar su eficacia en un $68 \%$, aplicando métodos como la respiración, sugestión verbal, yoga, hipnosis entre los más importantes. ${ }^{19}$

Ríos y Rodríguez en el 2014 y 2016 respectivamente, afirman que tratamientos farmacológicos han sido usados ante casos de ansiedad antes del tratamiento odontológico, y afirman que son el tratamiento de elección cuando los métodos no farmacológicos no han dado resultados o a su vez podrían ser combinados ya que esta consiste en una depresión menor de la conciencia, que mantiene la habilidad del paciente para respirar y de actuar de manera independiente respondiendo de forma apropiada a estimulaciones físicas y verbales. $^{22,42}$

\section{DISCUSIÒN}

La ansiedad y el miedo al tratamiento odontológico son un problema que han ido avanzando progresivamente especialmente en la población infantil, ya que los niños al llegar a la consulta odontológica traen consigo una carga emocional que expresan en el momento de su atención y está relacionada de acuerdo con su edad y nivel de madurez; la responsabilidad como profesionales no es solo limitarse a la prevención y a la salud de problemas bucodentales sino conocer cómo manejar al paciente ansioso para brindarle mejor estabilidad, tranquilidad y confort el momento de su atención dental. La ansiedad dental es una respuesta a situaciones de amenaza que ha ido avanzando con el tiempo, según Lazo en el 2010 encontró mayor prevalencia de ansiedad en el sexo masculino, que en el sexo femenino y en el estudio de Rivera en el 2005 concuerda con esta información, excepto en el estudio de Caycedo en el 2008, en el que hubo mayor prevalencia en las mujeres utilizando la escala de Corah. ${ }^{5,10,12}$

Según Ramos en el 2013 la ansiedad y miedo pueden influir en situaciones de atención y tratamiento dental y puede ser problemas de salud oral. Algunos estudios insisten que las niñas tienen más ansiedad y manifiestan mayor miedo que los niños pero de forma poco significativa y que esto depende más que todo de la madurez psicológica del niño. ${ }^{6}$

La edad es un factor que se encuentra íntimamente relacionado con los niveles de ansiedad y miedo en los niños; estudios han demostrado que los niños más pequeños manifiestan mayores niveles de ansiedad que los niños de mayor edad esto confirma el estudio de los autores que se mencionan anteriormente, confirmando que la edad es un factor importante a considerar. $6,12,28$

Según Lazo en el 2010 y Ciraiz y Luna en el 2015 respectivamente aseguran que la ansiedad es una respuesta 
Tabla 8. Efectividad de manejo y control de la ansiedad y miedo al odonólogo no farmacológicos.

\begin{tabular}{llc}
\hline Referencia & Tratamiento & Efectividad \\
\hline Bare (2004) ${ }^{(39)}$ & Música de fondo (MD) & $89 \%$ \\
Bare (2004) & Libros y revistas (MD) & $75 \%$ \\
Rojas (2011) ${ }^{(40)}$ & Percepción del Control & $40 \%$ \\
Lima (2008) ${ }^{(19)}$ & Técnicas de Relajación & $68 \%$ \\
Gómez(2012) ${ }^{(41)}$ & Musicoterapia & $72 \%$ \\
Rodríguez S. (2009) ${ }^{(42)}$ & Comunicación Verbal (DMH) & $65 \%$ \\
\hline
\end{tabular}

Tabla 9. MD: Medios de Distracción; DMH: Decir-Mostrar-Hacer

normal del niño de adaptación ya que esta recién explorando un medio desconocido que genera amenaza o algún tipo de peligro, y esa será su manera de protegerse, actuando de manera defensiva o con algún mal comportamiento. 5,26,27

\section{CONCLUSIÓN}

Al realizar esta revisión bibliográfica se pudo manifestar que la ansiedad y el miedo son dos fenómenos que van de la mano ya que causan aprehensión y dificultades al paciente en la consulta, se diferencian porque el miedo es una reacción inmediata ante algo que el paciente reconoce, la ansiedad al contrario es un estado en que el paciente identifica la amenaza; los factores asociados a esta ansiedad son varios entre los más destacados en la literatura son la sensación de vibración de las fresas en la pieza de mano, seguido de la actitud del dentista y de la impresión o ruido al instrumental odontológico son los principales factores causantes; se dice que la edad es un factor que interviene con este fenómeno especialmente en niños de 4-8 años de edad con una frecuencia de ansiedad y miedo moderado; para poder valorar o medir este problema se desarrollaron instrumentos el más utilizado para valorar la ansiedad y considerado como una escala de gold estándar es el C-DAS o escala de Corah y para valorar el miedo en niños podemos decir que el mejor manejado por los profesionales es el CFSS-DS o Children Fear Survey Scale.

Los profesionales de la salud tienen la responsabilidad de tener los conocimientos adecuados para el tratamiento de la ansiedad y miedo para asegurar una mejor calidad de vida del paciente en primera instancia en la consulta y también asegurar evitar problemas futuros, es así que se encontró que la musicoterapia o medios distractores son los tratamientos no farmacológicos de primera línea para prevenir y manejar en su momento la ansiedad y miedo al tratamiento dental, se podría recurrir a medios farmacológicos como la sedación consiente si es que estos no funcionan o se podría probar la utilización de los dos, siempre y cuando sean supervisados de la manera adecuada.

Conflicto de intereses y financiamiento Los autores declaran no tener conflicto de, haber cumplido con los requisitos de autoría y haber autofinanciado este artículo.

\section{Referencias}

1 Abanto J, Vidigal E, Carvalho T, Sá S, Bönecker M. Factors for determining dental anxiety in preschool children with severe dental caries. Braz. Oral Res. 2017; 31(13).

2 Najafpour E, Aminabadi N, Nuroloyuni S, Jamali Z, Shirazicorres $\mathrm{S}$. Can galvanic skin conductance be used as an objective indicator of children's anxiety in the dental setting? Clinical and Experimental Dentistry. 2017 Marzo; 9(3).

3 Albuquerque W, Corrêa M, Abanto J. Evaluación de la ansiedad materna en el tratamiento odontopediátrico utilizando la escala de Corah. Rev Estomatol Herediana. 2007;: p. 22-24.

4 Cirilo J, Córdova E, Marín Y, Robles, Siquero K, Díaz A. Nivel de ansiedad a los factores ambientales en dos servicios odontológicos públicos:hospitalario y universitario. Odontol. Sanmarquina. 2012; 15(1).

5 Lazo A. Nivel de ansiedad antes y después de un tratamiento odontológico en niños de 4 a 8 años de edad. Revista estomatológica de Altiplano. 2010; 2(12).

6 Ramos K. Niveles de ansiedad y miedo en niños en las clínicas de la facultad de odonotopediatría de la Universidad de Cartagena. Tesis de Grado. Cartagena: Universidad de Cartagena, Departamento de investigación; 2013. Report No.: ISSN.

7 Valdés JM. Caracterización de pacientes con miedo al estomatólogo. Acta Odontológica Colombiana. 2015 JulioDiciembre; 5(2): p. 33-46.

8 Rivero, Lima M, Casanova Y. Miedo, ansiedad y fobia al tratamiento estomatológico. Instituto Superior de Ciencias Médicas Carlos J. Finlay. 2016 Marzo; 1(1). 
9 Fonseca L, Sanchis C. Análisis comparativo entre las distintas escalas de valoración de comportamiento, Ansiedad 26 y Miedo Dental en Odontopediatría.Revisión Bibliográfica. Therapeía. 2013;: p. 81-95.

10 Caycedo C, Cortés O, Gama R, Rodríguez H. Ansiedad al tratamiento odontológico: características y diferencias de género. Red de Revistas Científicas de América Latina, el Caribe, España y Portugal. 2008 marzo; 15(1): p. 259-278.

11 Arrieta K, Díaz S, Verbel J. Factores asociados a sintomatología clínica de miedo y ansiedad en pacientes atendidos en Odontología. REV CLÍN MED FAM. 2013; 6(1): p. 17-24.

12 Rivera I. Ansiedad y miedos dentales en escolares hondureños. Revista Latinoamericana de Psicología. 2005; 37(3): p. 28 461-475.

13 Doerr, P Factor asociated with dental anxiety. JADA. 1998 Agosto; 129.

14 Moore R. Dentists' perceived stress and its relation to perceptions about anxious patients. Community Dent Oral 29 Epidemiol. 2001; 29: p. 73-80.

15 Moore R. Prevalence and characteristics of dental Anxiety 30 in Danish Adults. Community Dent Epidemiol. 1993; 21(6): p. 292-296.

16 Mcneil D, Helfer A, Weaver B, Graves R, Kyle B, Davis A. Memory of Pain and Anxiey Associated with tooth 3 extraction. J Dent Res. 2011; 90(2).

17 Crofts N, Brough E, Wilson K, Beddis A, Girdler N. Anxiety and Quality of life in Phobic Dental Patient. J Dent Res. 2010; 89(3): p. 302-306.

18 Elter J, Ronald P, Strauss , James D, Beck.. Assesing Dental Anxiety, Dental Care Use and Oral Status in Older Aldults. Jada. 1997; 128.

19 Lima M, Guerrier L, Toledo A. Técnicas de relajación en pacientes con ansiedad al tratamiento estomatologico. Rev Hum Med. 2008 Mayo-Dic; 8(2-3).

20 Ray J, Wide B, Boddin L, Bergreen U, Lichtenstein , Broberg.. Heritability of Dental Fear. J Dent Res. 2010; 89(3): p. 297-301.

21 Hmud R, Walsh L. Ansiedad dental: causas, complicaciones y métodos de manejo. Revista de menos Intervención en Odontología. 2009; 2(1).

22 Ríos M, Herrera A, Rojas G. Ansiedad dental: Evaluación y 36 tratamiento. Avances en Odontoestomatología. 2014; 30(1): p. 39-46.

23 Locker R, Poulton.. Psychological disorders and dental anxiety in a young adult population. Community Dent Oral 37 Epidemiol. 2001; 29(1).

24 Coric A. Dental fear and anxiety in older children: an association with parental dental anxiety and effective pain 38 coping strategies. Journal of Pain Research. 2014 Agosto; 4(7).

25 Tiwari N, Tiwari S, Thakur R, Agrawal, Shashikiran, S. S. 39 Bare L, Dundes L. Strategies for Combating Dental Anxiety. Evaluation of treatment related fear using a newly developed fear scale for children: "Fear assessment picture scale" and 40 its association with physiological response. Contemporary
Clinical Dentistry I. 2015 Julio; 6(3).

6 Ciraiza E. Nivel de ansiedad previo y posterior al tratamiento dental mediante el test de dibujos de Venham modificado en pacientes niño que asisten a la clinica dental de la facultad de odontología de la Universidad de San Carlos de Guatemala. Tesis de grado. Guatemala: Universidad de San Carlos de Guatemala, Departamento de Odontología; 2015.

27 Luna G. Evaluación del nivel de ansiedad en pacientes pediátricos que acuden a la facultad de odontología de la Universidad central del Ecuador mediante estimulación musical. Tesis de Grado. Quito: Universidad Central del Ecuador., Facultad de Odontología; 2015.

8 Tasayco R. Nivel de ansiedad en relación a la utilización de musicoterapia en niñios de 6 a 8 años atendidos en la clínica odontológica de Norbet,Wiener,Lima-Perú,2010. Tesis de posgrado. Lima-Perú: Universidad de Wiener, Facultad de Odontología; 2010.

29 Sunil R. Evalation Of Dental Fear in children during on dental Visit usinf children Fear Survey. Jaype. 2013; 6(1).

Singh P, Pandey R, Nagar K, Dutt K. Reliability and factor analysis of childrens fear survey schedule-dental subscale in India subjects. Journal of Indian Society of pedodontics and preventy Dentistry. 2010; 28: p. 151-155.

1 Howard K, Freeman R. Reliability and validity of a faces version of the Modified Child Dental Anxiety Scale. International Journal of Paediatric Dentistry. 2007; 17: p. 281288.

32 Buchanan H. Validation of a Facial Image Scale to assess child dental anxiety. International Journal of Paediatric Dentistry. 2002; 12: p. 47-52.

33 Bahammam M, Hassan M. Validity and reliability of an Arabic version of the modifid dental anxiety scale in Saudi adults. Saudi Med J. 2014; 35(11): p. 1384-1389.

34 Lalić M, Aleksić J, Milić, Malešević, Jovičić B. Reliability and validity of the Serbian version of Children's Dental Fear Questionnaire. Pubmed. 2015 Jul; 72(7): p. 602-7.

35 Tunc E, Firat D, Onur O, Sar V. Reliability and validity of the Modified Dental Anxiety Scale (MDAS) in a Turkish population. Community Dent Oral Epidemiol. 2005; 33: p. 62-357.

6 Majstorovic M, Veerkamp I, Skrinjaric. Reliability and validity of measures used in assessing dental anxiety in 5- to 15year-old Croatian children. European Journal of pediatric dentistry. 2003; 4: p. 197-202.

37 Lin C, Wu S, Yi C. Association between Anxiety and Pain in Dental treatment: A sysstematic. Review and Meta-analysis. Journal of Dental Research. 2017; 96(2).

8 Tijdschr N. [Dental treatment of fearful children: subjection, denyal or -guidance?]. Pubmed. 2017 Abril; 124(4): p. 215221. Journal of Dental Education. 2004 Agost; 68: p. 1172-1177.

0 Rojas C, Harwardt P, Sassenfeld A, Molina Y, Herrera, Ríos, et al. Eficacia de las técnicas de percepción de control y 
relajación en la reducción de ansiedad dental. Acta Odontológica Venezolana. 2011; 49(4).

41 Gómez R, Durán L, Cabra L, Pinzón C, Rodriguez L. Musicoterapia para el control de ansiedad odontológica en niños con síndrome de Downñ. Hacia la Promoción de la Salud. ; 17(2).

42 Rodriguez S, Pinto F, Alcocer J. Técnicas de atención y control de conducta en el paciente infantil. Clínica Odontológica de U Latina La Carpio. 2009; 14.

43 Rodriguez H. Tratamientos farmacológicos y no farmacológicos para la ansiedad al tratamiento estomatológico. Revista Cubana de Estomatología. 2016; 53(4).

Recibido: 20 de Noviembre de 2017

Aceptado: 15 de Enero de 2017 
